\title{
Systematic Study of Video Mining with Its Applications
}

\author{
Mallappa G. Mendagudli ${ }^{\mathrm{a}}$, K. G. Kharade ${ }^{\mathrm{b}}$, T. Nadana Ravishankar ${ }^{\mathrm{c}}$, K. Vengatesan ${ }^{\mathrm{d}}$ \\ ${ }^{a} B L D E A$ 's A.S.Patil College of Commerce, Vijayapur, Karnataka \\ ${ }^{b}$ Department of Computer Science, Shivaji University, Kolhapur, Maharashtra \\ 'Department of Computer Science \& Engineering, Vel Tech High Tech, Avadi, Chennai \\ ${ }^{d}$ Department of Computer Engineering, Sanjivani College of Engineering, Kopargaon
}

\begin{abstract}
Effective methods for video indexing will be more valuable as digital video data continues to grow. It has been years since we've seen this level of new multimedia research. The content analysis aims to create high-level descriptions and annotations by treating language and facts as data. Data mining is a technique that seeks out previously unknown facts and patterns in large datasets. A video can include several different kinds of data, such as images, visuals, audio, text, and additional metadata. Thanks to its broad application in various disciplines, like security, education, medicine, research, sports, and entertainment, it is often used differently. Data mining aims to discover and articulate exciting patterns that are hidden in a lot of video footage. While video mining is still in its infancy, data mining is more mature. A considerable amount of research must be done to turn the mined video into usable content
\end{abstract}

Keywords. Event Detection, Frequent Pattern, Video Classification, Video Mining, Video Processing

\section{Introduction}

The massive growth in multimedia databases has been fueled by the ongoing improvements in multimedia acquisition and storage technology. As computing power, sensors, and storage improve, multimedia data development increases. Multimedia data mining is the process of discovering patterns from a large set of previously unknown data. Implicit knowledge extraction deals with extracting non-explicit patterns hidden in the multimedia data or the relationships between the multimedia data [14]. In the non-structural nature of multimedia data, multimedia data management is among the essential tasks. An immediate threat is handling all the multimedia data, such as multimedia text, images, audio, and video, with an unnecessarily complicated structure such as multimedia text, images, audio, and video. Currently, video is widely accepted as one of the most prominent multimedia regarding information and entertainment. Video mining enables efficient browsing and harvesting of video information while also making video retrieval possible in a gigantic data store or the cloud. Video data comes in many different varieties [2].

\footnotetext{
${ }^{1}$ K. G. Kharade, Department of Computer Science, Shivaji University, Kolhapur Email:kgk_csd@unishivaji.ac.in
} 
There are two main types of videos: scripted and non-scripted. Produced videos follow a set script or plan, and are edited, compiled, and disseminated to viewers. Fiction movies, news stories are perfect demonstrations of pre-produced videos. The footage in non-scripted videos does not occur through an act of will, nor do they always happen in a predictable setting, such as surveillance footage, sports footage, and business meetings [4]. Non-scripted videos deal with occurrences of interest and highlights. While video mining does not extract everything about the video, it can also discover various patterns related to video structure, events, activities, and much more. Many different video mining techniques have been explored to extract meaningful information from video data [18]. Finding information in a highly compressed feature space is still a difficult task. Association mining, pattern detection, and clustering are video classifications referred to the following should be kept in mind when mining video;

a) It should be possible to solve the problem by applying relatively simple mathematical techniques

b) The implementation should be unsupervised

c) There should be some room for flexibility

d) It should display essential and significant events

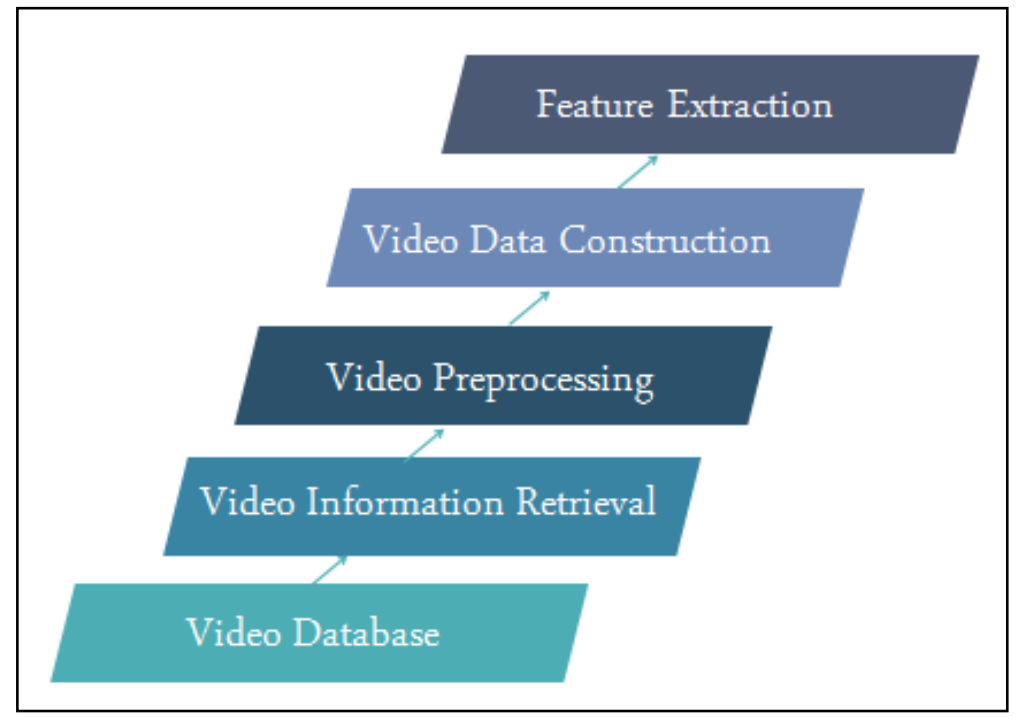

\section{Framework}

Fig. 1: Common Steps in Video Mining

Video data mining applies the principles of implicit knowledge discovery when seeking to discover information that has been encoded into the data sequence. It's a difficult task to visualize patterns in a video frame sequence, because video contains both complex structured and unstructured data. When patterns are identifiable in the database, the discovery process incorporates discovering patterns [21]. Video is made up of individual images strung together sequentially, and images are referred to as frames. A single-camera has only one shooting sequence, called a shot. They utilize a shot-based strategy, while the object-based approach does not. This transaction-level analysis is done at the item level, and around each image is examined as well. Objectbased representation makes content and indexing easier when it comes to search [5]. 
When doing object-based tracking, region-tracking and spatiotemporal segmentation are commonly employed. In the regular course of day-to-day life, we're interested in watching two kinds of videos: those containing structure and those that don't. When it comes to movies, videos, and news, these are all vehicles for carrying video content[17]. There is no video in the sports surveillance footage. Associated rule mining requires three steps: preliminary processing, association rule development, and database construction. To ensure that the video data is processed first, it should be first converted from its un-structural raw data format into a structured layout. Once the video data has been transformed, features are extracted to obtain the main features and stored in structured databases. By employing data mining methods, mining can be done with this generated output [15].

\section{Video Pre-Processing}

Most of the time, unstructured video data is a data source. No matter how hard you try, this knowledge cannot be duplicated. The video is broken up into individual video frames to convert video data files into a format in which it can be easily structured. The shot boundary is found in the first phase before any processing has taken place [6]. Video data is broken down into the shot, which is the basic unit. The primary components of video are text, audio, and video. Object segmentation techniques and color analysis are used to capture visual features. Both frequency-domain and timedomain audio features are included, and text features that use video text processing techniques. In the early stages of processing, all the various components are applied to process the raw video sequences into video clips that can be organized and sorted into time steps [7]. Video frames can be used as an input once the key frames have been extracted with the shot partitioning algorithm. Using multimodal features, time-lapse shots, the key frames are discovered, and in the next step, the video data is generated [16].

\section{Video Data Construction}

When it comes to creating a video sequence, it is not uncommon for every symbolic stream (visual, audio, text, and objects with frame window) to be assigned a symbol. Each frame in the video is treated as a time unit [13]. According to the Lookup Table (which maps every feature to a symbolic value), each time unit's extracted features are translated into extended streams to produce transformed elements at the end of the video. The temporal video sequence, which consists of multiple streams into a single stream, is made using the original video data. A transformed structured video sequence is utilized in the mine associations in the video database [1].

\section{Feature Extraction}

Visual features are extracted from the key frame images using image processing algorithms. Audio feature extraction systems use signal classification techniques to collect several audio segments from the input audio signal. It is possible to glean useful information from the text present in the video by conducting mining on the associations [8]. Videotext is divided into two categories: featured video and associated video. When the text is superimposed on video sequences, it helps the viewer know what is being presented. We apply a symbol the first time we find text within the data. Every step, procedure, and significant action is shown in step-by-step detail in a video [20]. 


\section{Applications Of Video Mining}

Many different applications, including defense, exploration, education, entertainment, and sports, use video mining technology. Video data mining is the search for knowledge to extract data from visual sources, establishing patterns and dynamics. The video mining options on this page might be of interest to you.

i)Vehicles monitoring procedures: Video is now an essential part of nearly every security system and can help you decide which cameras and monitoring setups you'll need to meet your needs. We provide a wide range of video recording, image management, as well as cutting-edge technology. Video data mining can be used to regulate various types of public transportation. Digital video processing is helpful for vehicle monitoring. Since it is a video-based system, the traffic department can track vehicles [9]. Traffic flow monitoring is done on major thoroughfares to keep transport speeds and tolls on schedule.

ii)Traffic control management: One of the new uses thermal imaging is discovering is on traffic video. Extractive analytics or mining for video data can lead to better decisions regarding traffic management Viewers will have access to the live feeds of traffic cameras installed on the traffic posts [12]. This real-time input is brought into the system to give it more data. According to the available video, the traffic management systems the procedures will be controlled by the controls. Due to this solution, such as a suspicious vehicle activity, the analytics dashboard is immediately notified [6].

iii)Health Monitoring: It's used as the method for gathering the observation data. Many experimental modal analyses have shown that there is effective when natural frequencies are used as measurements. A CCTV stream can give healthcare workers a window into the well-being of the patient's health. Eulerian video magnification and optical flow stream camera are excellent for tracking the infant's respiratory difficulties. Eliminated research will allow for better treatment options in the future [10].

iv)Automated Transcription Video Data: Due to transcribing, small businesses can take advantage of audio/video data mining; audio/video data mining offers excellent opportunities for gaining unstructured data. It results in accurate texts being transformed into an increase in business productivity and efficiency. Simultaneously, it runs various routines, including feedback analysis, demographics, and legal compliance [11].

v) Enhanced Security with Live Video Streaming: It is excellent for transcribing individual-level large small businesses because the audio/video/video mining of accurate texts improves productivity and effectiveness. It performs various analyses, demographics, including feedback, while it also operates in compliance with a legal regulation [19].

\section{Conclusion}

The application of data mining is known as data mining and describes processes that search for hidden patterns and knowledge in large volumes of data. Until relatively recently, much of data mining research has focused on alpha-numeric databases. It remains the case, as multimedia data mining has not received the same attention. This paper discusses the current state of video data mining and the challenges in the field. It would be best if you concentrated on addressing these issues to gather extensive amounts of valuable data from an abundance of video data. In the data mining realm, a tremendous amount of research and development has been done over the years. Low- 
levels out of spatial, inferring concepts from low-level videos and dealing with complexity in unstructured video unabstracted data.

\section{References}

[1] Chen, X., \& Zhang, C. (2006). An Interactive Semantic Video Mining and Retrieval PlatformApplication in Transportation Surveillance Video for Incident Detection. Sixth International Conference on Data Mining (ICDM'06), 129-138. https://doi.org/10.1109/ICDM.2006.20

[2] Kexue Dai, Jun Zhang, \&Guohui Li. (2006). Video Mining: Concepts, Approaches, and Applications. 2006 12th International Multi-Media Modelling Conference, 477-480. https://doi.org/10.1109/MMMC.2006.1651376

[3] Kharade, K. G., Kharade, S. K., \& Kumbhar, V. S. (2017). A Comparative Study of Traditional Server and Azure Server. Journal of Advances in Science and Technology, 13(1), 329-331.

[4] Potharaju, S. P., \& Sreedevi, M. (2017). A Novel Clustering Based Candidate Feature Selection Framework Using Correlation Coefficient for Improving Classification Performance. Journal of Engineering Science \& Technology Review, 10(6).

[5] Katkar, S. V., Kharade, K. G., Kharade, S. K., \& Kamat, R. K. (2020). An Intelligent Way of Modeling and Simulation of WO3 for Supercapacitor. In N. Thapa, Recent Studies in Mathematics and Computer Science (pp. 109-117). Hooghly, West Bengal, India: Book Publisher International.

[6] Kharade, K. G., Kamat, R. K., \& Kharade, S. K. (2017). Review On Microsoft Windows Azure Technology. National conference on "Innovative Trends in Electronics and Allied Technology (ITEAT-2017), (p. 33).

[7] Kharade, K. G., Kamat, R. K., \& Kharade, S. K. (2017). Utilization of Copy Logger Software Package to Protect Sensitive Data from Malicious Individuals. National conference on "Innovative Trends in Electronics and Allied Technology (ITEAT-2017)" organized by Yashvantrao Chavan Institute of Science, Satara, (p. 19).

[8] Kharade, K. G., Kamat, R. K., Kharade, S. K., \&Katkar, S. V. (2019). Automation of Paper Setting Process to Improve Effectiveness of The Examination System of The University. Journal of Emerging Technologies and Innovative Research,490-493.

[9] Kharade, K. G., Kamat, R. K., Mudholkar, R. R., \& Kharade, S. K. (2018). Removable Drive Blocker Application for Virus Detection. International Journal of Research Culture Society, 233-234.

[10] Kharade, K. G., Kharade, S. K., \&Kumbhar, V. S. (2018). Impact of Digital India on Various Sectors. Indian Journal of Innovation in Management and Excellence In Research (IJIMER), 37-40.

[11] Kharade, K. G., Kharade, S. K., \&Oza, K. S. (2018). Security Threats In E-commerce. Indian Journal of Innovation In Management And Excellence In Research (IJIMER), 14-17.

[12] Patil, B. P., Kharade, K. G., \&Kamat, R. K. (2020). Investigation on Data Security Threats \& Solutions. International Journal of Innovative Science and Research Technology, 5(1), 79-83.

[13] Rao, G., Kumari, K., Shankar, D., \& Kharade, K. G. (2021). A comparative study of augmented realitybased head-worn display devices. Materials Today: Proceedings. 1-9.

[14] Li, X., Shi, M., \& Wang, X. (Shane). (2019). Video mining: Measuring visual information using automatic methods. International Journal of Research in Marketing, 36(2), 216-231. https://doi.org/10.1016/j.jiresmar.2019.02.004

[15] Matsuo, Y., Amano, M., \& Uehara, K. (2002). Mining video editing rules in video streams. Proceedings of the Tenth ACM International Conference on Multimedia - MULTIMEDIA '02, 255. https://doi.org/10.1145/641007.641058

[16] Othman, E. H., Abderrahim, G., \& Jaber, E. B. (2017). Mining Moocs Videos Metadata Using Classification Techniques. Proceedings of the 2nd International Conference on Big Data, Cloud and Applications, 1-6. https://doi.org/10.1145/3090354.3090450

[17] Porreca, A., Scozzari, F., \& Di Nicola, M. (2020). Using text mining and sentiment analysis to analyze YouTube Italian videos concerning vaccination. BMC Public Health, 20(1), 259. https://doi.org/10.1186/s12889-020-8342-4

[18] Rosenfeld, A., Doermann, D., \&DeMenthon, D. (Eds.). (2003). Video Mining. Springer US. https://doi.org/10.1007/978-1-4757-6928-9

[19] Potharaju, S. P., Sreedevi, M., \& Amiripalli, S. S. (2019). An Ensemble Feature Selection Framework of Sonar Targets Using Symmetrical Uncertainty and Multi-Layer Perceptron (SU-MLP). In Cognitive Informatics and Soft Computing (pp. 247-256). Springer, Singapore.

[20] Vijayakumar, V., \&Nedunchezhian, R. (2012). A study on video data mining. International Journal of Multimedia Information Retrieval, 1(3), 153-172. https://doi.org/10.1007/s13735-012-0016-2 
[21] Santhosha, S. \& Kiran, N.. (2018). A Systematic Review on Existing Data Mining Approaches Envisioned for Knowledge Discovery from Multimedia. International Journal of Electrical and Computer Engineering. 81. 908-916. 10.11591/ijece.v8i2.pp908-916. 